\title{
Fine architecture of the fascial planes around the lateral femoral cutaneous nerve at its pelvic exit: an epoxy sheet plastination and confocal microscopy study
}

\author{
Zhaoyang Xu, MB, MMed, ${ }^{1,2}$ Lili Tu, MB, MMed, ${ }^{1}$ Yanyan Zheng, MB, ${ }^{3}$ Xiaohui Ma, MB, ${ }^{1}$ Han Zhang, ${ }^{4}$ \\ and Ming Zhang, MB, MMed, PhD²
} Departments of ${ }^{1}$ Anatomy and ${ }^{3}$ Ultrasound, Anhui Medical University, Hefei, China; and ${ }^{2}$ Department of Anatomy and ${ }^{4}$ School of
Medicine, University of Otago, Dunedin, New Zealand

\begin{abstract}
OBJECTIVE Meralgia paresthetica is commonly caused by mechanical entrapment of the lateral femoral cutaneous nerve (LFCN). The entrapment often occurs at the site where the nerve exits the pelvis. Its optimal surgical management remains to be established, partly because the fine architecture of the fascial planes around the LFCN has not been elucidated. The aim of this study was to define the fascial configuration around the LFCN at its pelvic exit.

METHODS Thirty-six cadavers ( 18 female, 18 male; age range 38-97 years) were used for dissection ( 57 sides of 30 cadavers) and sheet plastination and confocal microscopy ( 2 transverse and 4 sagittal sets of slices from 6 cadavers). Thirty-four healthy volunteers (19 female, 15 male; age range 20-62 years) were examined with ultrasonography.

RESULTS The LFCN exited the pelvis via a tendinous canal within the internal oblique-iliac fascia septum and then ran in an adipose compartment between the sartorius and iliolata ligaments inferior to the anterior superior iliac spine (ASIS). The iliolata ligaments newly defined and termed in this study were 2-3 curtain strip-like structures which attached to the ASIS superiorly, were interwoven with the fascia lata inferomedially, and continued laterally as skin ligaments anchoring to the skin. Between the sartorius and tensor fasciae latae, the LFCN ran in a longitudinal ligamental canal bordered by the iliolata ligaments.
\end{abstract}

CONCLUSIONS This study demonstrated that 1) the pelvic exit of the LFCN is within the internal oblique aponeurosis and 2) the iliolata ligaments form the part of the fascia lata over the LFCN and upper sartorius. These results indicate that the internal oblique-iliac fascia septum and iliolata ligaments may make the LFCN susceptible to mechanical entrapment near the ASIS. To surgically decompress the LFCN, it may be necessary to incise the oblique aponeurosis and iliac fascia medial to the LFCN tendinous canal and to free the iliolata ligaments from the ASIS.

https://thejns.org/doi/abs/10.3171/2018.7.JNS181596

KEYWORDS anatomy; fascial configuration; lateral femoral cutaneous nerve; meralgia paresthetica; pelvic exit; sheet plastination; peripheral nerve

$\mathrm{M}$ ERALGIA paresthetica (MP) is characterized by symptoms of pain, numbness, itching, and paresthesia at the anterolateral thigh and is commonly caused by mechanical entrapment of the lateral femoral cutaneous nerve (LFCN).,13,21 It is commonly believed that mechanical entrapment of the LFCN occurs at the site where the nerve exits the pelvis. ${ }^{2}$ A number of anatomical studies have discovered different variations in the LFCN exiting the pelvis, but the most common pattern was medial to the anterior superior iliac spine (ASIS). ${ }^{26}$

Of those structures surrounding the LFCN medial to the ASIS, an occasional fascial canal was reported at the level of the inguinal ligament, ${ }^{3,4}$ whereas a more consistent fat-filled flat tunnel was localized within the fascia lata between the sartorius and the tensor fasciae latae. ${ }^{19,31}$ Hanna argued that the LFCN ran in its own canal, with a fascial plane completely surrounding the nerve from the level of the inguinal ligament to the thigh. ${ }^{8}$ Based on this anatomical concept, most recently, he reported on a case series of 19 patients with MP who underwent $360^{\circ}$ decompression of the LFCN at its pelvic exit, in which special attention was paid to the fascia deep to the LFCN. ${ }^{9}$ 

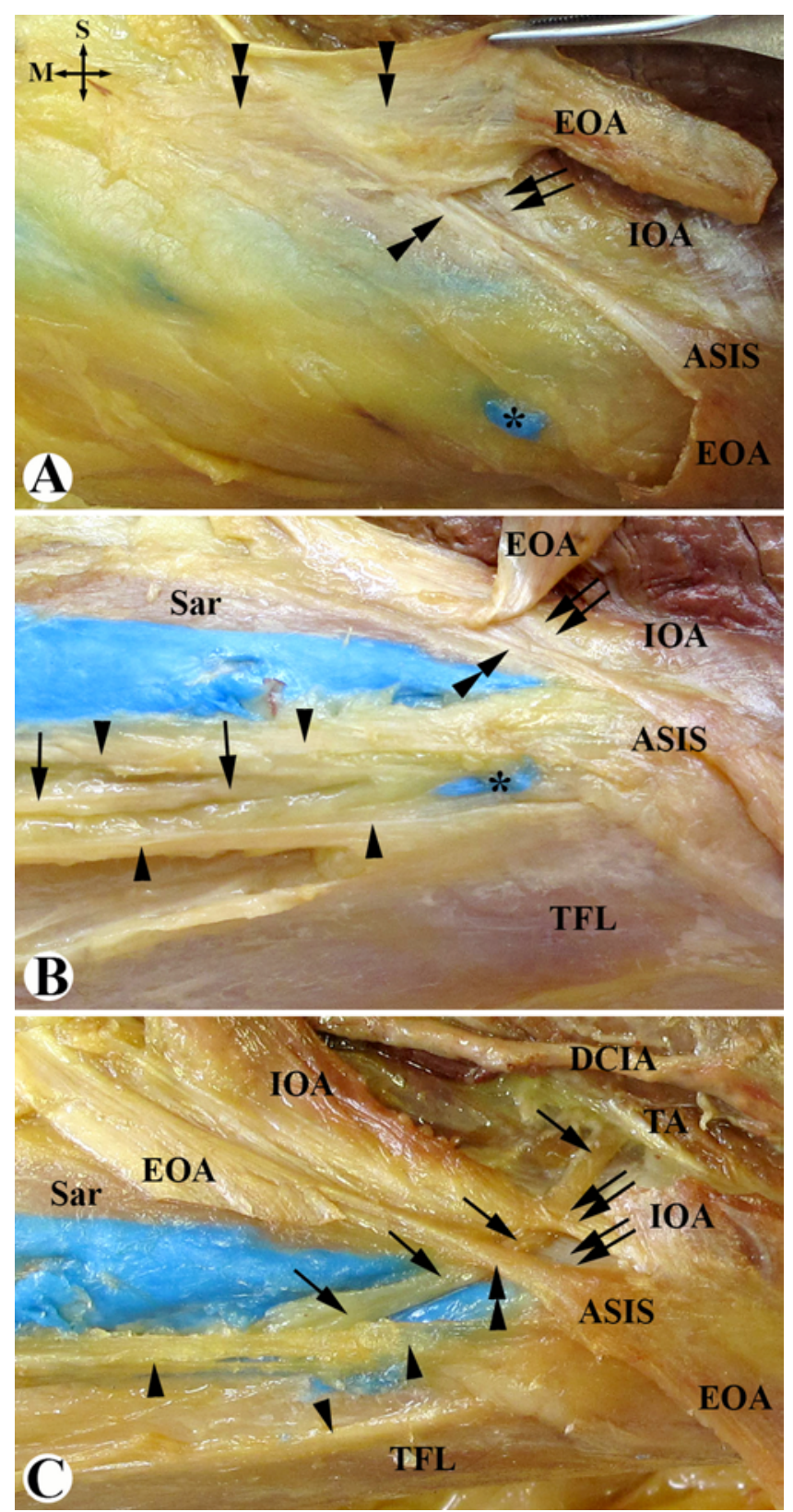

FIG. 1. Layer-by-layer dissection of the inguinal and upper thigh region after blue latex injection (asterisk) into the sartorial fascia. A: Aponeurotic fibers (double arrowheads) of the external oblique abdominis (EOA) either parallel to the internal oblique abdominis (IOA; double arrows) or inferiorly extending to the fascia lata. The EOA is partially cut and reflected inferiorly. The crossed arrows indicate the orientation ( $\mathrm{S}=$ superior; $\mathrm{M}=$ medial). $\mathbf{B}$ : The ligamental canal bordered by iliolata ligaments (single arrowheads), as termed in this study. Single arrows indicate the LFCN. Sar = sartorius muscle (partially covered by the injected blue latex, indicated by asterisk); TFL = tensor fasciae latae. C: The tendinous canal within the aponeurotic fibers of the IOA (double arrows) and under the EOA (double arrowheads). DCIA = deep circumflex iliac artery; $\mathrm{TA}=$ transversus abdominis.

A number of surgical treatments for MP have been employed, including simple decompression, a combination of superficial and deep decompressions, decompression with iliac fascia incision, medial transposition, and $360^{\circ}$ decompression, $, 1,5,6,9-11,15$ and some of them have provided very good results, with success rates ranging from $93 \%$ to $100 \% .{ }^{18,23,24}$ However, the efficacy and underlying mechanism of individual surgical techniques remain to be pinpointed, mainly because of lack of understanding of the anatomy of the fascial planes around the LFCN. ,, $69-11^{-1}$

Technically, the study of the fascial architecture is complicated by the fact that great difficulties exist in dissecting out delicate structures. Although histological examination may be able to overcome the problem, the application of such a method is greatly limited by the size of sample areas, alteration of tissue architecture during decalcification, and difficulty in tracing the origin of a fibrous structure. These laboratory limitations are largely overcome by the newly developed technology of sheet plastination, which not only ensures preservation of the in situ position of bone, cartilage, and soft tissues without decalcification but also allows these structures to be examined undisturbed in their natural state at both macroscopic and microscopic levels. ${ }^{16,28-30}$ The aim of this study was to use sheet plastination technology combined with confocal microscopy to define the fascial configuration around the LFCN at its pelvic exit.

\section{Methods}

A total of 36 cadavers (18 female, 18 male; age range 38-97 years) and 34 healthy volunteers (19 female, 15 male; age range 20-62 years) were studied. None of the healthy volunteers had any history of thigh or pelvic injury or surgery. The cadavers assigned to this project were bequeathed for medical education and research purposes with the written informed consent from the donor or the next of kin as mandated under the Human Tissue Act. This study was performed in accordance with our institutional ethical guidelines and approved by the institutional ethics committees.

\section{Dissection}

In 30 cadavers (57 sides), the LFCN and its surrounding structures were exposed via layer-by-layer dissection. The intersections between the LFCN and the inguinal ligament or the lateral border of the sartorius were identified; the distances between the intersections and between the ASIS and the intersection were measured. Three cadavers were injected with blue latex underneath the fascia of the sartorius muscle prior to dissection (Fig. 1A and B).

\section{Epoxy Sheet Plastination and Confocal Microscopy}

Six cadavers ( 2 female, 4 male; age range $46-87$ years) were prepared as transverse (2 sets) and sagittal (4 sets) plastinated slices. Sheet plastination is a modern, anatomical technique in which water and fat within tissues and cells are replaced by durable, long-lasting, transparent resin, thus preserving all the structures in situ in their natural state, suitable for examination at both macroscopic (Fig. $2 \mathrm{~A}, \mathrm{~B}$, and C) and microscopic (Fig. 2a, b, and c) levels. ${ }^{28,30}$ The plastination procedure was performed as previously described..$^{30}$ In brief, the specimen was frozen at $-80^{\circ} \mathrm{C}$ for 7 days and cut into serial sections. The thickness of the section was $2.5 \mathrm{~mm}$, and the interval between adja- 

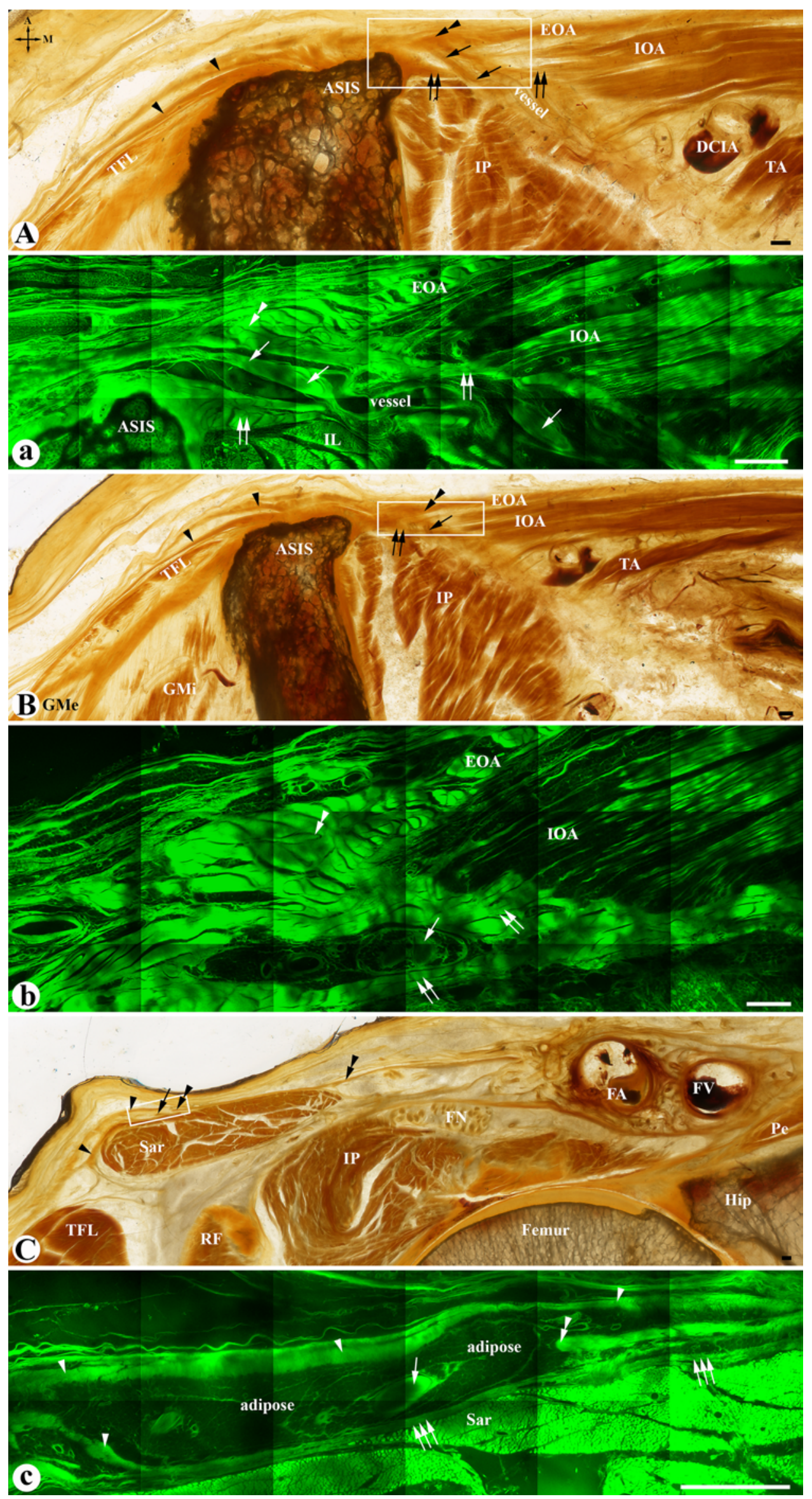

J Neurosurg Volume 131 • December 2019
FIG. 2. Three adjacent transverse plastinated sections at the levels of the anterior superior iliac spine (ASIS) (A and $B$ ) and the upper thigh (C). Panels $a, b$, and $c$ are the confocal images corresponding to the area shown in the boxes in A, B, and C. Single arrows indicate the LFCN; double arrows, the aponeurotic fibers of the internal oblique abdominis (IOA); triple arrows, the epimysium of the sartorius muscle (Sar); double arrowheads, the aponeurotic fibers of the external oblique abdominis (EOA); and single arrowheads, the iliolata ligaments. $\mathrm{DCI} A=$ deep circumflex iliac artery; $F A=$ femoral artery: $\mathrm{FN}=$ femoral vein; $\mathrm{GMe}=$ gluteus medius; $\mathrm{GMi}=$ gluteus minimus; $\mathrm{IP}=$ iliopsoas muscle; $\mathrm{Pe}=$ pectineus; $\mathrm{RF}=$ rectus femoris; $\mathrm{TA}=$ transversus abdominis; $\mathrm{TFL}=$ tensor fasciae latae. The crossed arrows indicate the orientation $(A=$ anterior; $M=$ medial). Bar $=1 \mathrm{~mm}$. 
cent sections was $0.9 \mathrm{~mm}$. The sections were dehydrated in $-30^{\circ} \mathrm{C}$ acetone for 4 weeks, followed by degreasing in acetone at $22^{\circ} \mathrm{C}-24^{\circ} \mathrm{C}$ for 3 weeks, then impregnated with an epoxy resin mixture of E12/E1/AE10/AE30 (Biodur) at $0^{\circ} \mathrm{C}$ for 2 days, and cured at $45^{\circ} \mathrm{C}$ for 5 days. The prepared sections were examined under a Leica MZ8 stereoscopic, dissecting microscope (Leica). The plastination process results in collagen, elastin, myofilaments, and neurofilaments being endogenously auto-fluorescent at the 488-nm excitation; thus, relevant plastinated sections were examined under a confocal laser scanning microscope (Nikon). The thickness of the optical section was set at $16.7 \mu \mathrm{m}$ under a $10 \times$ objective and all images were electronically recorded and montaged.

\section{Three-Dimensional Model of the LFCN Course Near the ASIS}

Forty-two images from one set of the plastinated sections were assembled. The LFCN and its surrounding structures near the ASIS were manually segmented as 2D images, then reconstructed and displayed as 3D images in Thermo Scientific Amira Software (Amira 6.5.0, Thermo Fisher Scientific).

\section{Ultrasound Scan}

Ultrasound images were obtained with a Siemens Acuson Antares machine (Siemens Medical Solutions) with a Siemens vfx13-5 linear ultrasound transducer. The subject was in a supine position, and the ultrasound transducer was placed in a transverse position around the ASIS. The ASIS, iliopsoas muscle, internal and external abdominis muscles, sartorius muscle, and tensor fasciae latae were used as landmarks to identify and trace the LFCN.

\section{Results}

In the cadavers examined in this study, the LFCN coursed medially and inferiorly to the ASIS, exited the pelvis, and entered into the thigh (Figs. 1 and 2).

\section{The Tendinous Canal}

Within the pelvis, the LFCN was underneath the iliac fascia (Figs. 1C and 2A). At the level medial to the ASIS and posterior to the external oblique abdominis, it pierced a fibrous septum of the iliac fascia and aponeurotic fibers of the internal oblique abdominis (Figs. 1C, 2A, and 2a). The LFCN traversed within the iliac fascia and the aponeurotic fibers of the internal oblique abdominis, which formed a rigid fully enclosed tendinous canal (Figs. 2B, $2 \mathrm{~b}$, and $3 \mathrm{~A}$ ). The tendinous canal was posteroinferior to the aponeurotic fibrous bundles of the external oblique abdominis or the inguinal ligament (Figs. 1C, 2a, and 2b).

\section{The Adipose Compartment and "lliolata Ligaments"}

Inferiorly to the ASIS, the superficial aponeurotic fibers of the external oblique abdominis extended inferiorly to the thigh and contributed to the fascia lata (Figs. 1A and $3 \mathrm{~B}$ ). The inferior aponeurotic extension of the external oblique abdominis covered the medial part of the sartorius muscle and had a free lateral border inferior to the ASIS
(Figs. 2C and c). After exiting the tendinous canal (Fig. 3B), the LFCN passed under the inferior aponeurotic extension of the external oblique abdominis and entered into an adipose compartment, which was sandwiched between the fascia lata and the epimysium of the sartorius muscle (Figs. 1C, 2C, 2c, and 3B-D). This part of the fascia lata was formed by $2-3$ curtain strip-like ligaments which were termed the "iliolata ligaments" in this study. Superiorly, the iliolata ligaments inserted to the ASIS (Figs. 1B, 1C, 2A, 2B, 3C, and 3D); inferiorly (Fig. 3C and D) and medially (Fig. $2 \mathrm{C}$ and $\mathrm{c}$ ), they contributed to the fascia lata; and laterally, they fanned out and continued as skin ligaments in the subcutaneous tissue (Fig. 2A and B).

\section{The Ligamental Canal}

The LFCN coursed along the adipose compartment inferolaterally (Figs. 1C, 2C, and 2c), pierced one of the iliolata ligaments along the lateral border of the sartorius muscle (Fig. 1B), and ran in a longitudinal ligamental canal, which was between the iliolata ligaments and medial to the tensor fasciae latae (Figs. 1B and 3D).

\section{Localization of the Tendinous and Ligamental Canals}

The mean distances $( \pm \mathrm{SD})$ from the ASIS to the point where the LFCN crossed the inguinal ligament or pierced the iliolata ligament along the lateral border of the sartorius muscle were $2.15 \pm 0.94 \mathrm{~cm}$ and $5.07 \pm 1.59 \mathrm{~cm}$, respectively. The mean value of the angle between 2 lines from the ASIS to the cross-points was $40^{\circ} \pm 23^{\circ}$.

\section{Ultrasonography Evaluation}

The course of the LFCN around its pelvic exit was evaluated in 34 healthy volunteers. On the transverse sonogram, the LFCN appeared as a hypoechoic round or elliptical structure with hyperechoic round or linear signals within it. In the pelvis, the LFCN ran within hyperechoic signals over the iliacus muscle. The hyperechoic signals had a clear continuation medially with the transverse and internal oblique abdominis muscles and laterally passed under the external oblique aponeurosis and inserted to the ASIS (Figs. 4A and B). At the level of the tendinous canal, the nerve was within the internal oblique aponeurotic fibers and medial to the sartorius and under the external oblique aponeurotic fibers (Fig. 4C). At the level of the ligamental canal, the nerve was sandwiched between the iliolata ligaments, lateral to the sartorius and medial to the tensor fasciae latae (Fig. 4D). The iliolata ligaments and the LFCN were better visualized on a transverse sonogram view (Fig. 4D).

\section{Discussion}

This study revealed the fibrous configuration of the perineural fascial sheath of the LFCN at its pelvic exit, identified the origin and insertion of these fibers, and traced their course. Figure 5 is a 3D image generated from one set of the plastinated slices (Fig. 5A) and illustrates the principal findings of this study. 1) The LFCN exits the pelvis via a tendinous canal within the internal obliqueiliac fascia septum (Fig. 5B and C). 2) Inferiorly to the ASIS, the LFCN runs in an adipose compartment over the 


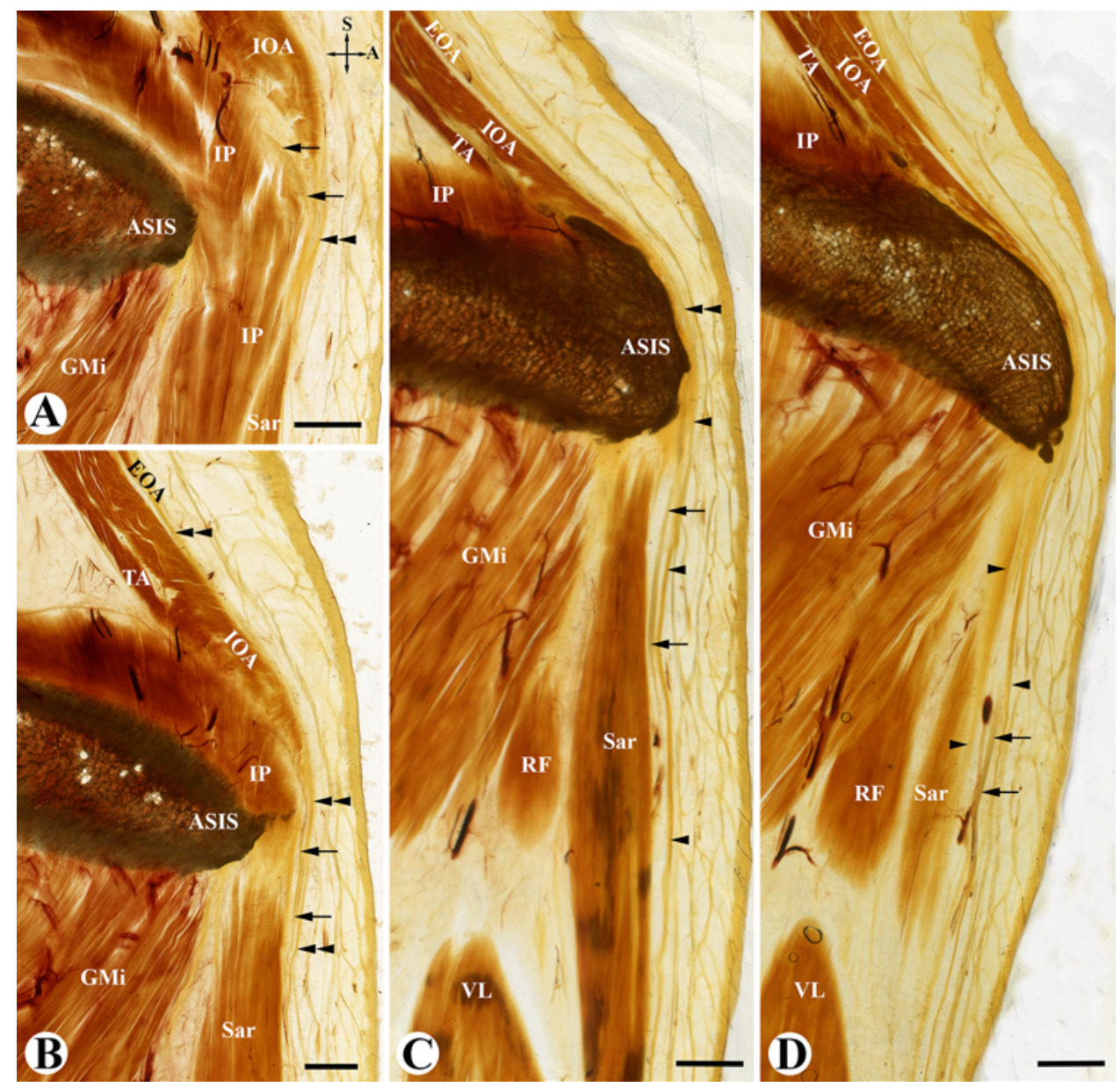

FIG. 3. Four adjacent sagittal plastinated sections from medial to lateral (A-D) at the level of the ASIS. Single arrows indicate the LFCN; double arrowheads, aponeurotic fibers of the external oblique abdominis (EOA); and single arrowheads, the iliolata ligaments. $\mathrm{GMi}=$ gluteus minimus; IOA = internal oblique abdominis; IP = iliopsoas muscle; RF = rectus femoris; Sar = sartorius muscle; $\mathrm{TA}=$ transversus abdominis; $\mathrm{TFL}=$ tensor fasciae latae; $\mathrm{VL}=$ vastus lateralis. The crossed arrows indicate the orientation ( $\mathrm{S}=$ superior; $\mathrm{A}=$ anterior). $\mathrm{Bar}=10 \mathrm{~mm}$.

epimysium of the sartorius muscle and under the inferior extension of the external oblique aponeurosis (Fig. 5D) and the curtain strip-like iliolata ligaments, which contribute to the fascia lata (Fig. 5E). 3) Between the sartorius and tensor fasciae latae, the LFCN runs in a longitudinal ligamental canal bordered by the iliolata ligaments (Fig. $5 \mathrm{E}$ and $\mathrm{F}$ ).

\section{The Tendinous Canal of the LFCN}

Although variability of the LFCN course near the ASIS has been extensively reported, ${ }^{2,17,22,27}$ it has been commonly accepted that the LFCN exits the pelvis under or through the inguinal ligament. ${ }^{26}$ Gray's anatomy ${ }^{25}$ states that the inguinal ligament is the inrolled and rounded lower border of the external oblique aponeurosis and its inferior extension is continuous with the fascia lata. The iliac fascia and internal oblique aponeurosis form a common septum (or iliopubic tract) between the inguinal ligament and the hip bone. ${ }^{25}$ Therefore, the LFCN must pierce the internal oblique-iliac fascia septum to exit the pelvis.
The present study confirmed this assumption and revealed that the septum was located deep and internal to the external oblique aponeurotic fibers and was dominated by the internal oblique aponeurotic fibers, which fused and intermingled with the iliac fascia and inserted to the ASIS (Fig. 5C). The LFCN pierced the septum, forming a tendinous canal, and then passed under the external oblique aponeurosis, including its inferior extension.

The tendinous canal identified in this study is different from previous descriptions of the LFCN as going through a tunnel or narrow canal between 2 "slips of attachment" from the inguinal ligament. ${ }^{14,15}$ The present study demonstrated that the tendinous canal was fully encircled by the horizontally oriented fibers from the internal oblique and iliac fascia. In addition to the internal oblique, the iliaca fascia was also reinforced by the transverse abdominis aponeurosis. Therefore, the abdominal muscles, particularly the internal oblique and transversus abdominis, must have a significant impact on the tendinous canal because the stretching of these muscles may tighten and narrow the canal. 

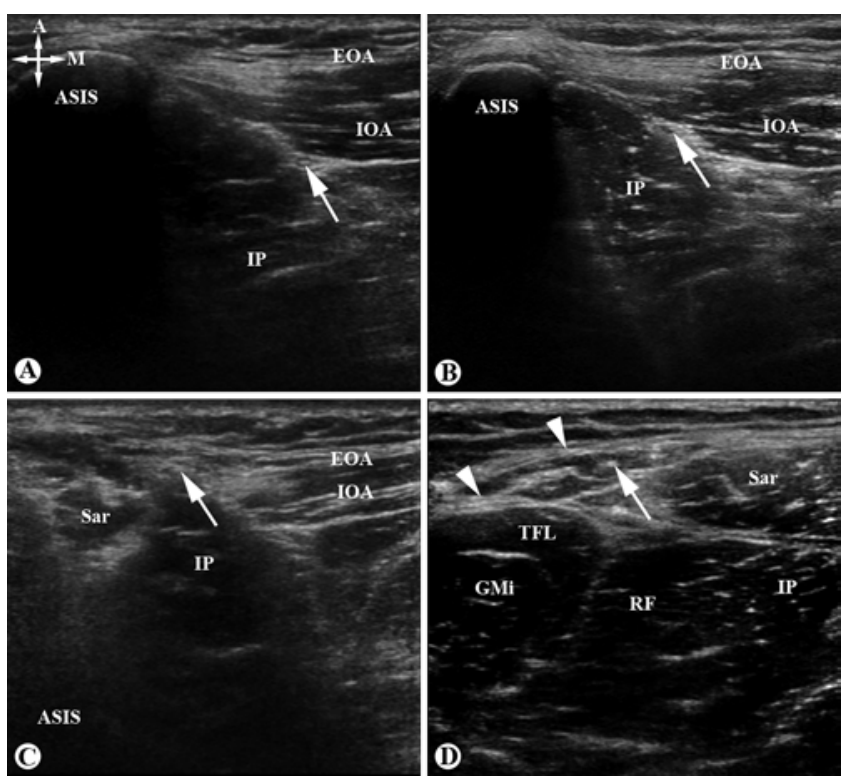

FIG. 4. Transverse sonogram views of the LFCN within the tendinous canal $(\mathbf{A}-\mathbf{C})$ and ligamental canal (D). The LFCN (arrows) appears as a hypoechoic round or elliptical structure with hyperechoic round or linear signals within it. Arrowheads indicate the iliolata ligaments. EOA = external oblique abdominis; $\mathrm{GMi}=$ gluteus minimus; $1 \mathrm{OA}$ = internal oblique abdominis; IP = iliopsoas muscle; RF = rectus femoris; Sar = sartorius muscle; TFL = tensor fasciae latae. The crossed arrows indicate the orientation ( $\mathrm{A}=$ anterior; $\mathrm{M}=$ medial).

\section{Iliolata Ligaments and the Ligamental Canal of the LFCN}

Using epoxy sheet plastination and confocal microscopy techniques to identify and trace fibrous origin and configuration, the present study revealed that the part of the fascia lata inferior to the ASIS was composed of the iliolata ligaments and the inferior extension of the un-inrolled superficial fibers of the external oblique aponeurosis. The iliolata ligaments were 2-3 curtain strip-like ligamental structures which superiorly inserted to the ASIS, inferiorly and medially interwoven with other parts of the fascia lata, e.g., the inferior extension of the inguinal ligament, and laterally fanned out and continued as skin ligaments in the subcutaneous tissue of the thigh. The iliolata ligaments may have been represented as those multiple layers of the fascia lata in the previous reports. ${ }^{19,20}$

Most recently, Hanna reported that a complete fascial canal surrounded the LFCN, from the inguinal ligament to the terminal branches of the nerve in the thigh. ${ }^{8}$ The present study detailed the fibrous configuration of Hanna's fascial canal of the LFCN. On the medial side of the sartorius, after piercing the tendinous canal, the LFCN ran between the epimysium of the muscle and the inferior extension of the inguinal ligament. On the lateral side of the sartorius, the nerve was between the epimysium of the muscle and the iliolata ligaments. As one of the iliolata ligaments intermingled with the epimysium of the sartorius along the lateral border of the muscle, the LFCN pierced the ligament and entered a longitudinal lenticular canal which was bordered by the iliolata ligaments, filled with fat and termed as the ligamental canal of the LFCN in this study. Thus, the LFCN fascial canal defined by Han- na may actually consist of 3 segments: a tendinous canal within the internal oblique-iliac fascia septum, an adipose compartment over the sartorius muscle, and a ligamental canal between the iliolata ligaments.

\section{Theoretical Surgical Strategy for LFCN Decompression}

One of surgical treatments for MP is to decompress the entrapped LFCN. In addition to neurectomy, several strategies have been suggested for decompression, including simple decompression, combination of superficial and deep decompressions, decompression with the iliac fascia incision, and medial transposition. ${ }^{5,6,9-11,15}$ To date, there is no consensus as to what is being decompressed in actuality. 5,10 The lack of understanding of the precise anatomy of the fascial configuration around the nerve appears to be part of the current debate on the optimal management strategy. ${ }^{9}$

The present study precisely defined that the internal oblique-iliac fascia septum and iliolata ligaments were 2 major anatomical structures that may make the LFCN susceptible to mechanical entrapment near the ASIS. Therefore, based on their fibrous origin and configuration, we propose a theoretical strategy for decompression of the LFCN. The main aims are 2-fold: to eliminate the muscular stretching of the tendinous canal medial to the ASIS and to diminish the tension of iliolata ligaments inferior to the ASIS. The key surgical technical points to pinpoint the potential mechanical entrapment sites are 1) to incise the internal and external oblique aponeurosis and iliac fascia medial to the tendinous canal of the LFCN in order to relax the tension generated from the abdominal muscles and 2) to free the iliolata ligaments from the ASIS. The technique of the $360^{\circ}$ decompression of the LFCN at its pelvic exit demonstrated by Hanna, in which the aponeurotic fibers of the tendinous canal were fully cut, would appear to meet these criteria. ${ }^{9}$ A slightly lateral extension to cut the iliolata ligaments can be considered for a further modification of the $360^{\circ}$ decompression technique, as it may further diminish the tension generated by iliolata ligaments (or fascia lata).

Ultrasound evaluation for the LFCN localization has been mainly focused on its segment in the adipose compartment over the sartorius and in the ligamental canal.9,12,31 For example, on both transverse and longitudinal ultrasound views, Hanna demonstrated the LFCN in the ligamental canal, which was bordered by the iliolata ligaments. ${ }^{8}$ Since the pelvic exit of the LFCN was at the internal oblique-iliac fascia septum, we performed ultrasound evaluation specifically at this spot in the present study.

The results indicate that using the internal and external oblique muscles, sartorius muscle, and ASIS as the landmarks, the tendinous canal can be identified and visualized with ultrasound. Three technical recommendations are 1) to use the quantitative data provided in this study to surface-mark the LFCN (Fig. 5F), (2) to scan along the external (or inguinal ligament) and internal oblique muscles laterally to the ASIS, and (3) to identify the LFCN inferomedial to the ASIS, deep to the inguinal ligament and within the internal oblique aponeurosis.

\section{Limitations}

This study has at least 2 limitations. First, the number 

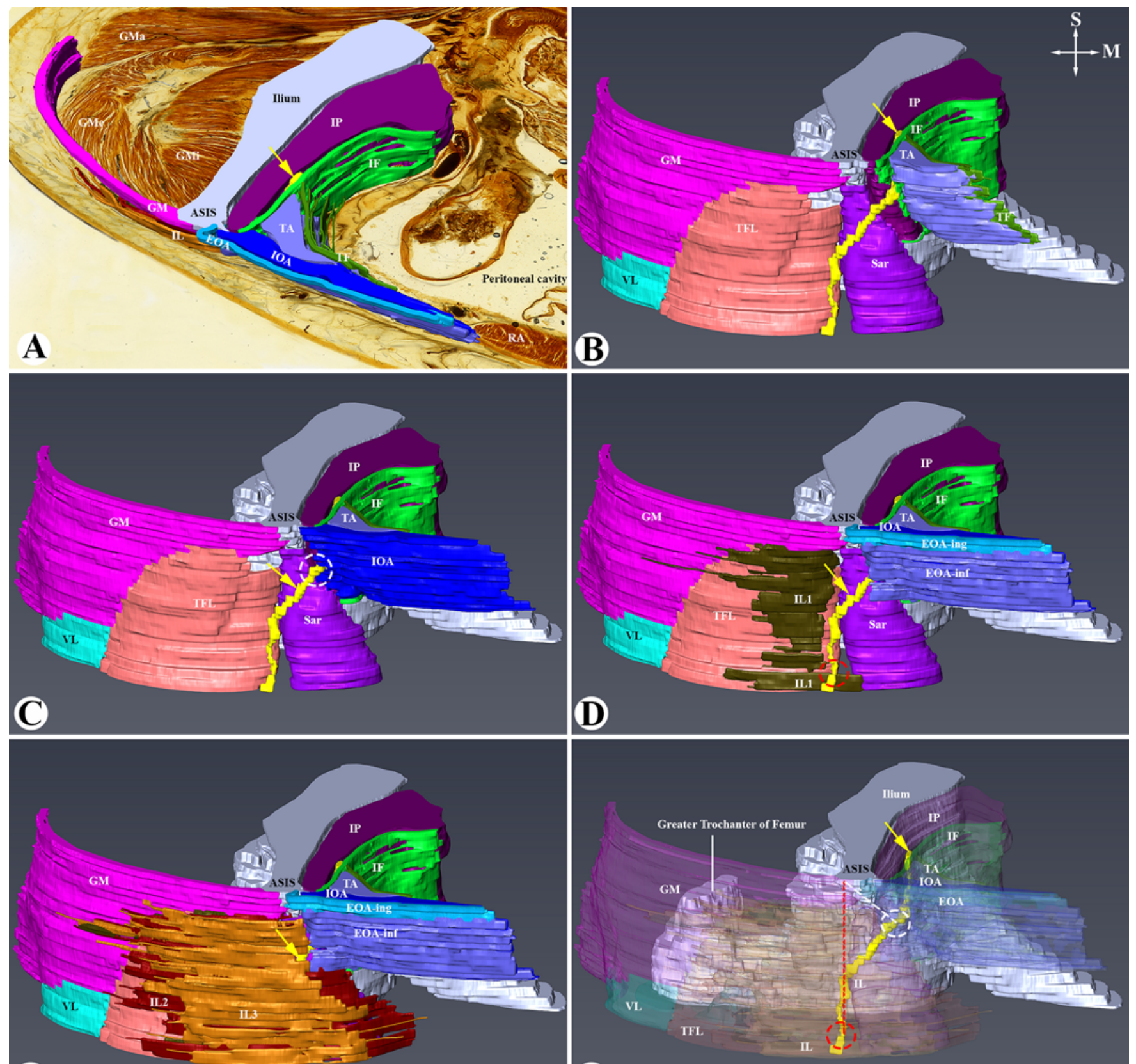

E

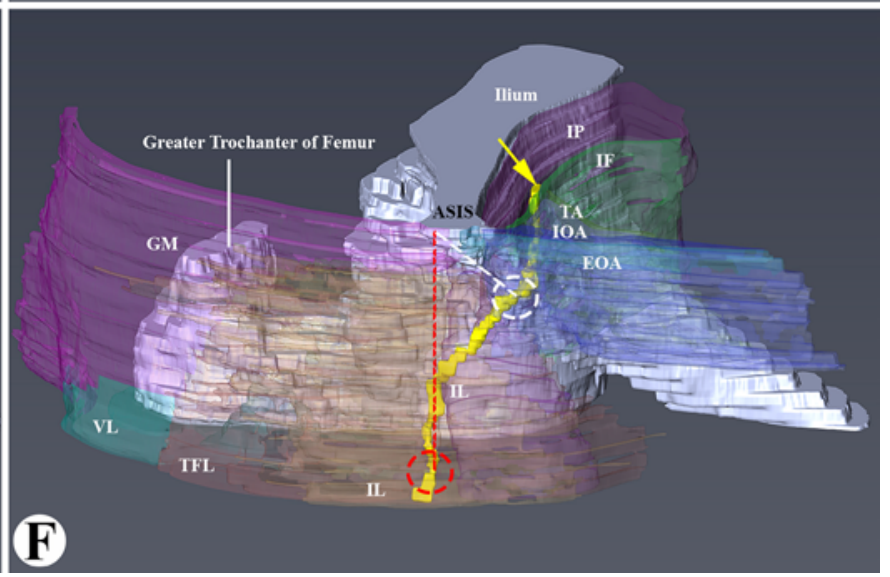

FIG. 5. 3D images reconstructed from one set of the plastinated sections showing the course of the LFCN (yellow arrows) and its neighboring structures near the ASIS. A: 3D image with 2D image of a plastinated section. EOA = external oblique abdominis; GM = tendinous sheet of gluteal muscles; $\mathrm{GMa}=$ gluteus maximus; $\mathrm{GMe}=$ gluteus medius; $\mathrm{GMi}=$ gluteus minimus; $\mathrm{IF}=$ iliac fascia; $\mathrm{IL}$ = iliolata ligaments; IOA = internal oblique abdominis; IP = iliopsoas muscle; RA = rectus abdominis; TA = transversus abdominis; TF = transversalis fascia. B: The intrapelvic LFCN between the iliopsoas muscle and iliac fascia. The iliac fascia is continuous with the transversalis fascia and reinforced by the tendinous fibers of the transversus abdominis. Sar = sartorius muscle; TFL = tensor fasciae latae; $\mathrm{VL}=$ vastus lateralis. The crossed arrows indicate the orientation ( $\mathrm{S}=$ superior; $\mathrm{M}=$ medial). $\mathrm{C}$ : The LFCN pierces the internal oblique-iliac fascia septum (white dashed circle) and enters the adipose compartment on the sartorius muscle. D and E: The LFCN runs over the sartorius muscle and under the inferior extension of the external oblique aponeurosis (EOA-inf) or iliolata ligaments (IL1) and enters into the ligamental canal between the iliolata ligaments (IL1, IL2, and IL3). EOA-ing = inguinal ligament part of the external oblique aponeurosis. F: Soft tissues surrounding the LFCN are illustrated translucently to demonstrate the entrance points of the tendinous (white dashed circle) and ligamental (red dashed circle) canals. The distances from the ASIS to the entrance of the tendinous (white dashed line) and ligamental (red dashed line) canals are about $2 \mathrm{~cm}$ and $5 \mathrm{~cm}$, respectively, and the angle between the 2 lines is approximately $40^{\circ}$. 
of the cadavers for the epoxy sheet plastination was small. The pattern of fibrous configuration revealed in this study may vary in different anatomical variations of the LFCN course, such as when the nerve rides over the ASIS or iliac crest. ${ }^{8,15,18}$ Second, care should be taken to clinically confirm the feasibility and efficacy of the theoretical surgical strategy for the LFCN decompression proposed in this study, although there is some indirect evidence to support the proposal. For example, at the level of the inguinal ligament, increasing evidence has shown that the patients experienced better results when deep decompression with cutting the fascia underlying the nerve is added to superficial decompression. ${ }^{5,9,18,23}$ At the level of the iliolata ligaments, clinical improvement was observed even in patients with surgery for MP where no LFCN was found, ${ }^{3}$ suggesting that the nerve may have been decompressed by surgical "blind" dissection of the iliolata ligaments.

\section{Conclusions}

This study defined that the pelvic exit of the LFCN was within the internal oblique aponeurosis and the iliolata ligaments form the part of the fascia lata over the LFCN and upper sartorius. The results suggest that the internal oblique-iliac fascia septum and iliolata ligaments may make the LFCN susceptible to mechanical entrapment near the ASIS. To surgically decompress the LFCN, it may be necessary to incise the oblique aponeurosis and iliac fascia medial to the LFCN tendinous canal and to free the iliolata ligaments from the ASIS.

\section{Acknowledgments}

We thank Marlene Black, Andrew McNaughton, and Johann Zwirner from the Department of Anatomy, University of Otago, for their plastination, confocal microscopy, and ultrasonography technical support. The project was funded by a University of Otago Research Grant (2018-20) and the National Natural Science Foundation of China (reference no.: 81671368). Z.X. is supported by a $\mathrm{PhD}$ scholarship, University of Otago. L.T. is supported by the Academic Overseas Training Program, Anhui Medical University.

\section{References}

1. Aldrich EF, van den Heever CM: Suprainguinal ligament approach for surgical treatment of meralgia paresthetica. Technical note. J Neurosurg 70:492-494, 1989

2. Aszmann OC, Dellon ES, Dellon AL: Anatomical course of the lateral femoral cutaneous nerve and its susceptibility to compression and injury. Plast Reconstr Surg 100:600-604, 1997

3. Carai A, Fenu G, Sechi E, Crotti FM, Montella A: Anatomical variability of the lateral femoral cutaneous nerve: findings from a surgical series. Clin Anat 22:365-370, 2009

4. Cheatham SW, Kolber MJ, Salamh PA: Meralgia paresthetica: a review of the literature. Int J Sports Phys Ther 8:883-893, 2013

5. de Ruiter GCW, Kloet F: Anatomical considerations on transposition of the lateral femoral cutaneous nerve. Clin Anat [epub ahead of print], 2018

6. de Ruiter GCW, Wurzer JAL, Kloet A: Decision making in the surgical treatment of meralgia paresthetica: neurolysis versus neurectomy. Acta Neurochir (Wien) 154:1765-1772, 2012

7. Dias Filho LC, Valença MM, Guimarães Filho FA, Medeiros RC, Silva RA, Morais MG, et al: Lateral femoral cutaneous neuralgia: an anatomical insight. Clin Anat 16:309-316, 2003

8. Hanna A: The lateral femoral cutaneous nerve canal. J Neurosurg 126:972-978, 2017

9. Hanna A: Transposition of the lateral femoral cutaneous nerve. J Neurosurg [epub ahead of print April 13, 2018; DOI: 10.3171/2017.8.JNS171120]

10. Hanna A, Hanna B: Response to: Anatomical considerations on transposition of the lateral femoral cutaneous nerve. Clin Anat [epub ahead of print], 2018

11. Hanna AS: Lateral femoral cutaneous nerve transposition: renaissance of an old concept in the light of new anatomy. Clin Anat 30:409-412, 2017

12. Hanna AS, Ehlers ME, Lee KS: Preoperative ultrasoundguided wire localization of the lateral femoral cutaneous nerve. Oper Neurosurg (Hagerstown) 13:402-408, 2017

13. Ivins GK: Meralgia paresthetica, the elusive diagnosis: clinical experience with 14 adult patients. Ann Surg 232:281286,2000

14. Jefferson D, Eames RA: Subclinical entrapment of the lateral femoral cutaneous nerve: an autopsy study. Muscle Nerve 2:145-154, 1979

15. Keegan JJ, Holyoke EA: Meralgia paresthetica. An anatomical and surgical study. J Neurosurg 19:341-345, 1962

16. Liugan M, Xu Z, Zhang M: Reduced free communication of the subarachnoid space within the optic canal in the human. Am J Ophthalmol 179:25-31, 2017

17. Majkrzak A, Johnston J, Kacey D, Zeller J: Variability of the lateral femoral cutaneous nerve: An anatomic basis for planning safe surgical approaches. Clin Anat 23:304-311, 2010

18. Nahabedian MY, Dellon AL: Meralgia paresthetica: etiology, diagnosis, and outcome of surgical decompression. Ann Plast Surg 35:590-594, 1995

19. Nielsen TD, Moriggl B, Barckman J, Kølsen-Petersen JA, Søballe K, Børglum J, et al: The lateral femoral cutaneous nerve: description of the sensory territory and a novel ultrasound-guided nerve block technique. Reg Anesth Pain Med 43:357-366, 2018

20. Patijn J, Mekhail N, Hayek S, Lataster A, van Kleef M, Van Zundert J: Meralgia paresthetica. Pain Pract 11:302-308, 2011

21. Pearce JM: Meralgia paraesthetica (Bernhardt-Roth syndrome). J Neurol Neurosurg Psychiatry 77:84, 2006

22. Ropars M, Morandi X, Huten D, Thomazeau H, Berton E, Darnault P: Anatomical study of the lateral femoral cutaneous nerve with special reference to minimally invasive anterior approach for total hip replacement. Surg Radiol Anat 31:199-204, 2009

23. Siu TL, Chandran KN: Neurolysis for meralgia paresthetica: an operative series of 45 cases. Surg Neurol 63:19-23, 2005

24. Son BC, Kim DR, Kim IS, Hong JT, Sung JH, Lee SW: Neurolysis for meralgia paresthetica. J Korean Neurosurg Soc 51:363-366, 2012

25. Standring S: Gray's Anatomy: The Anatomical Basis of Clinic Practice, ed 40. London: Churchill Livingstone Elsevier, 2008, pp 1064, 1351-1352, 1382

26. Tomaszewski KA, Popieluszko P, Henry BM, Roy J, Sanna B, Kijek MR, et al: The surgical anatomy of the lateral femoral cutaneous nerve in the inguinal region: a meta-analysis. Hernia 20:649-657, 2016

27. Üzel M, Akkin SM, Tanyeli E, Koebke J: Relationships of the lateral femoral cutaneous nerve to bony landmarks. Clin Orthop Relat Res 469:2605-2611, 2011

28. Xu Z, Chapuis PH, Bokey L, Zhang M: Denonvilliers' fascia in men: a sheet plastination and confocal microscopy study of the prerectal space and the presence of an optimal anterior plane when mobilizing the rectum for cancer. Colorectal Dis 20:236-242, 2017 
29. Xu Z, Chapuis PH, Bokey L, Zhang M: Nature and architecture of the puboprostatic ligament: a macro- and microscopic cadaveric study using epoxy sheet plastination. Urology 110:263.e1-263.e8, 2017

30. Zhang M, An PC: Liliequist's membrane is a fold of the arachnoid mater: study using sheet plastination and scanning electron microscopy. Neurosurgery 47:902-909, 2000

31. Zhu J, Zhao Y, Liu F, Huang Y, Shao J, Hu B: Ultrasound of the lateral femoral cutaneous nerve in asymptomatic adults. BMC Musculoskelet Disord 13:227, 2012

\section{Disclosures}

The authors report no conflict of interest concerning the materials or methods used in this study or the findings specified in this paper.

\section{Author Contributions}

Conception and design: M Zhang, Xu. Acquisition of data: Xu, Tu, Zheng, Ma. Analysis and interpretation of data: Xu, Zheng,
Ma, H Zhang. Drafting the article: M Zhang, Xu. Critically revising the article: $\mathrm{H}$ Zhang. Reviewed submitted version of manuscript: M Zhang, H Zhang. Approved the final version of the manuscript on behalf of all authors: M Zhang. Study supervision: M Zhang.

\section{Supplementary Information}

Previous Presentations

Portions of this work were presented in abstract form as proceedings at the 14th Annual Conference of Australian and New Zealand Association of Clinical Anatomists, Auckland, New Zealand, December 4-6, 2017, and at the 5th International Fascia Research Congress, Berlin, Germany, November 14-15, 2018.

\section{Correspondence}

Ming Zhang: University of Otago, Dunedin, New Zealand. ming. zhang@anatomy.otago.ac.nz. 